\title{
Physical training in prevention of seasickness
}

\author{
Bogdan Łokucijewski ${ }^{1}{ }_{\text {ABCDEFG }}$
}

\author{
${ }^{1}$ Department of Marine and Hyperbaric Medicine, Military Institute of Medicine
}

\section{Summary}

The paper discusses the results of the author's experiment aimed at determining the effects of physical exercises, particularly rapid changes of head position, on susceptibility to motion sickness. Navy sailors underwent trainings followed by test cruises under real circumstances. The method was found to be of about $50 \%$ effectiveness; intensive physical training alleviated the complaints during cruises in about half of the study population.

Key words: motion, seasickness, physical training

Seasickness creates many difficulties in training and performing daily duties of mariners who are mainly sensitive to this type of acceleration that occurs in sea conditions. Long-term training of seamen and cadets who are not able to adapt to such conditions or do not have the opportunity to acquire the adaptation due to the short sailing experience consequently cause large material loss of training institutions. It is also a waste of time for trainee and incurs the danger for people working together in a team and often dependent on the proper fulfilment of the relevant task of a seriously seasick crew member.

Prevention of seasickness can be passive or active, or it may combine both of these two features. Passive prevention involves taking into account the construction of the ship or its parts, components and devices that reduce inclining at rough seas. The devices such as the keel, gyroscopic sides, Frahm's tanks, hanging bunks on Kardan's joints (in passenger liners) must be mentioned here. None of these methods do not give satisfactory protection against the seasickness. Moreover, they are costly and occupy a lot of space inside the unit.

Active prevention includes agents and actions to reduce human reactions to stressful environmental factors, in this case for linear and angular acceleration, stimulating the vestibular inner ear analyzer. Medicines of various types and diverse effects are concerned here ( antihistaminica, sedativa, parasympatolytica). They are used preventively. What is more, physical training and specialized exercises imitating, to a lesser or greater extent ,unusual conditions of Earth's acceleration, found in the sea in stormy conditions. It will be extended later in this work.

The idea of "toughening on the land” is not new and has been known for over a hundred years, but despite numerous attempts to the issue it has not been resolved in a satisfactory manner until now. „Such training involves the gradual habituation of the balance organs to work with the exception of some false information coming from the periphery” (Wożżowa) or "the support for the creation of new pathways in brain, which to a lesser or greater extent, prevent the influx of afferent stimuli by prior operating routes - to neurons inducing reaction „(Claser). Here are two definitions of determining the theoretical assumptions of the methods used.
It has been observed for a long time that people who are professionally involved in physical activities requiring a sudden movement of the head or maintaining a delicate balance (acrobats, skaters, dancers, gymnasts) get sick less often. It was confirmed in the field (storm) and assessed the neuro-vegetative responses to the stimulation of the vestibule in the laboratory.

Among many studies in this field (1-5) only a few of them made in aviation (Ałandoroa) and the Soviet Navy (Okuniew) will be mentioned. Systematic conduct of specialized physical exercises in a month time decreased vestibular reactions in $37 \%$ of the patients. Combining exercises with a swing on a swing parallel raised the score to $65 \%$, while the use of Barany'ego chairs and the swings - up to $75 \%$.

In 1958 Okuniew (5) examined 70-membered group of sailors. During 3 months time they performed a special morning exercises, on which we largely based our current set of exercises. Training of the crew lowered susceptibility to artificial wave by $10 \%$ and the author concluded that the active training is not enough and must be supplemented by a passive workout, specifically stimulating the vestibular analyzer.

General conclusion from these studies emphasized that specifically targeted and properly conducted exercises not only positively affect the overall health of mariners, but also reduce their sensitivity to the acceleration. Doctors working on ships ship unfairly undermine the importance of the vestibular training that can be performed even in simple marine conditions. The effectiveness of the recommended exercises has not yet been clearly confirmed, but - as is rightly written by Wożżowa - if they contain elements of various accelerations which are adequate factor that tease vestibular analyzer - it can be assumed that habituation will influence the reaction of vestibular (or controlled by the vestibule) well in natural sea conditions.

Habituation is a gradual decrease in the reaction as a result of the repetition of a specific stimulus. This is not equiv to an adaptation of the receptor, which means localized and rapid loss of excitability as a result of its continuous stimulation (Glaser).

If on the way of the massive use of training Soviet researchers conclude - stands the effort or the difficulty of reconciling the order of the day, it can be limi- 
ted to certain members of the crew that are difficult to replace, people who are specially trained or whom have failed other methods of seasickness prevention.

The study presents a summary of two stages of the experiment. The first was carried out in 6 weeks time in 1966 on 60 mariners who worked on the same ship, of which 30 were a training (study) group with Oksywski Physical Fitness Centre and 30 respondents in a control group. Both groups were selected randomly and they were not accustomed to sea conditions. Training consisted of daily (scrupulously respected) performance of a set of special exercises and swings on the swing parallel.

These exercises would be described closer in the second stage of the study. The control of gained immunity was made during the sea cruise on „KT Breeze”, which lasted six hours on the $3^{\circ} \mathrm{B}$ sea state. It is more than enough for this type of unit for the development of seasickness in individuals with average susceptibility.

Both groups were placed on the ship in such a way that all respondents had similar social and living conditions (eye contact with the environment, the possibility to communicate) and were subjected to similar type and intensity of accelerations. The failure of fulfilment of these conditions was a serious allegation against some works written in the United States in recent years, and the results were found to be unreliable if they lacked information about how to deploy two groups, or when the control group was amidships and trained in the areas of bow or vice versa .

The results of the first stage are shown in Table I. the service and other activities and a period of intensive military training is troublesome and requires a goodwill of commanders at the various levels and considerable effort of a person who conducts and supervises the training. They all should be thanked.

Training, as in the first stage, consisted of a set of exercises performed for 15 minutes (the active part) and also 15-minute swinging on a specially constructed parallel (the passive part).

Physical exercises consisted of: running without moving forward (about $40 \mathrm{sec}$.), circulation of the head from left to right (40 times), left and right hands circulation (15 times), circulation of the body (30 times), backward and forward head bands (20 times), back and front flips together (3-5 times), squats (20 times), body bands with left and right circulation; after every 3 circulations quick snap of body and resting for two seconds; the same the other way round; in total 15 turns in each direction. In addition, skips on toes and cartwheels (3-5 in each direction). As a requirement there was the degree of mariner's physical fitness that must ensure correct and safe execution of the exercises.

The officer conducting the classes noted in the training diary the presence of the respondents and described the symptoms that occurred in the course of training. The rule was that the participants had to be currently healthy. Symptoms of the illness that occurred before the training excluded them. The most common complaints reported during the swings were nausea, headache, dizziness, malaise. In the first phase of the experiment (in Oksywie) there also was repeated vomiting observed. Generally, the symptoms were similar to those occurring while being seasick and having other motion sickness. Initially, these symptoms occurred in $42 \%$ of patients and their number was highest in the

Table I

\begin{tabular}{|l|c|}
\hline \multicolumn{2}{|c|}{ Training group (30 people) } \\
\hline $\begin{array}{l}\text { a) Complaints during the training } \\
\text { b) Complaints during the cruise }\end{array}$ & $\begin{array}{c}-14 \text { people }(46 \%) \\
-8 \text { people }(26 \%)\end{array}$ \\
\hline \multicolumn{2}{|c|}{ Control group (30 people) } \\
\hline \multicolumn{2}{|c|}{-16 people $(53 \%)$} \\
\hline a) Complaints during the cruise
\end{tabular}

The fact that both homogeneous groups of mariners differed only by the conducted training increased by about $50 \%$ in the control group, it was considered an encouraging result for further research and it was proposed to carry out the second stage of a study on a larger group of people in Ustka.

Such studies were conducted in the summer 1972. There were 100 healthy mariners aged 19-21 who had not been floating selected for this purpose. The control group had also consisted of 100 mariners the same age as the study group remaining under similar conditions and having no sea experience.

To begin with, it should be noted that the conduct of the 45-minute workout every day for 6 weeks in such a large group in a way that would not interfere first days of training and decreased gradually with the habituation to the repeated stimuli. Ten of the most sensitive respondents reported subjective symptoms almost to the end of the training. As time passed, these symptoms were getting lighter and after a month there were no longer, apart from an occasional vomiting, dizziness and nausea.

The next day after the training the control cruise was conducted, in which 100 people from the study group and the control group took part. The groups were located on two the same class ships and were under the conditions described above and the trip was organised in the same time. The cruise lasted six hours and took place at the $3^{\circ} \mathrm{B}$ state of the sea, which was enough to develop the symptoms in sensitive individuals, but - be- 
cause of class of the ships - we found these conditions too mild and it did not allow us to differentiate clearly both groups. Due to the course of service and ship cruises the study could not be repeated.

Respondents' mood was checked every 30-minutes by the personnel who did not know the division into groups, which ensured in a further way objectivity of the subjects' assessment. The results were jotted down into the individual questionnaires. To make writing the results down easier, the complaints were divided into 4 groups depending on the severity of the condition. There were: "0" - no signs, no subjective symptoms, good mood; "1" - mild nausea, lack of appetite, pallor, perspiration; "2" - severe nausea, periodic vomiting, dizziness or headache; "3" - repeated vomiting.

From the study group during the voyage $20 \%$ of respondents became ill, including 15 people who have previously reported various ailments during training and 5 people who previously felt good. The individuals who had the worst symptoms while swinging, had also more severe seasickness.

In the control group symptoms of varying intensity were observed in $48 \%$ of patients. In general, the symptoms were more severe than in the training group. The number of people suffering in this group was almost equal to the number of people feeling not well while swinging - in the study group. Therefore, it can be assumed that both groups had similar susceptibility to seasickness, which has been differentiated as a result of training in favour of the study group.

Thus the training was the probable cause of reducing the vulnerability of trained people. This data is shown in Table II.

Table II
After substituting the pattern with the results from tables I and II we receive the effectiveness of the method of $54 \%$. This figure is similar to the ratings obtained during the study of drugs to prevent seasickness and which is considered to be effective. "Efficiency" of commonly known dramamine is assessed on the basis of the above formula to about $50 \%$, and regarded as the best drug hyoscine of $60-80 \%$. Rating largely depends on the voyage, on which the experimenter has no influence if you do not use the man-made waves in swimming pools with well-known parameters (2). Under mild conditions "the effectiveness" is estimated to be very high, in heavy too low.

The results of the experiment conducted in a total number of 260 mariners seem to indicate that specialized physical exercises combined with a swing on a swing parallel can be assumed as one of the methods to adapt to the conditions in the sea a selected group of average people. The method is useful, and therefore the training can be carried out in winter, ie. in the period that is rare to sail across the sea, and when there are objective difficulties of habituation in natural conditions, so what descriptively defines the Russian word: "omoriaczywanije".

In addition, such training can be used not only for habituation, but also in individual cases to select mariners to serve on ships. Those sailors who had problems during the entire training cycle sould not be directed to the sea service, but refer them to a specialist examination in Navy Hospital. The others, with a moderate susceptibility, who as a result of training, experience less symptoms, can in order to maintain habituation, exer-

\begin{tabular}{|l|l|}
\hline \multicolumn{2}{|c|}{ Training group (100 people) } \\
\hline $\begin{array}{l}\text { a) Complaints during the training } \\
\text { b) Complaints during the cruise }\end{array}$ & $-15 \%$ \\
& $-20 \%$ \\
\hline \multicolumn{2}{|c|}{ Control group (100 people) } \\
\hline a) Complaints during the cruise & $-48 \%$ \\
\hline
\end{tabular}

It is difficult to give sufficiently objective assessment of the effectiveness of the methods used in the prevention of seasickness. Simplifying the training can be treated as the equivalent of "medicine" and the control group as a "placebo" and use the formula proposed by Chinna (1) and commonly used to assess the efficacy of drugs (preventive) in seasickness. This formula below determines the amount of sensitive respondents (both groups are equally vulnerable, selected randomly), who did not become ill because of the use of a "medicine" (in this case training):

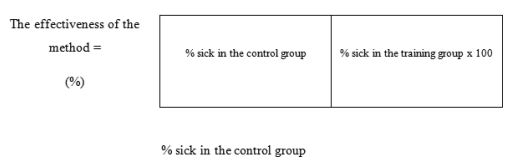

cise twice a week at PE classes for the entire period of training on land.

The main objection for the method of habituation on land is a mismatch used incentives to stimuli encountered later in the sea conditions. We recently showed that the exposure to vestibular stimuli is too small to cause motion sickness, and decrease sensitivity to the same stimulus but of a greater intensity (Graybiel).

The discussion about a particularly stressful stimuli in seasickness has not been definitively completed, although the vast majority of researchers in favour of a decisive role in the acceleration of linear motion sickness (swing parallel). Although, habituation regard to specific vestibular stimulus, and thus acquired for one type of movement does not necessarily apply to other 
(Money), but the existence of the transfer and the use of different stimuli at the same time allows for habituation of similar types of movement. However, transfer does not occur in the same extent as in the case of medicines used in the prevention of motion sickness (Graybiel).

However, this method leads to habituation by repeating the exercises combining both types of acceleration, so accustom to inhibit excess stimuli resulting from an unusual body position relative to the ground, should cause a gradual reduction in the overall response for those stimuli that are not common in everyday life. The existence of transfer shows that the habituation process occurs mainly in the central nervous system, but the role of the peripheral vestibular system in this process is not clear.

\section{References}

1. Chinn H.J. Motion sickness. USAF Rept: 1951.

2. Glaser E.M. The physiological basis of habituation. London; Oxford University Press: 1966.

3. Graybiel A. Aerospace Med, 1965, 36:733.

4. Money K.F. Physiol.Rev. 1970, 50:1.

5. Wożżowa A.I., Okuniew R.A.: Ukacziwanije i borba o nim. Medicina, Leningrad 1964.

\section{Correspondence address:}

Łokucijewski Bogdan MD PhD

Department of Marine and Hyperbaric Medicine, Military Institute of Medicine

ul. Grudzińskiego 4

81-103 Gdynia 3 